\title{
Versichertenkarte: die Haltung der FMH
}

\section{Judith Wagner}

a Judith Wagner leitet die neue Stabsstelle e-Health im FMHGeneralsekretariat.

Aus Gründen der Lesbarkeit wird im folgenden auf die weibliche Form verzichtet.
Korrespondenz: Judith Wagner FMH Elfenstrasse 18 CH-3000 Bern 15
Mit der Verabschiedung von Artikel 42a KVG (siehe Kasten) im Parlament wurde die Grundlage für die Einführung der sogenannten «Versichertenkarte» gelegt. Das Bundesamt für Gesundheit hat nun die Verordnungsbestimmungen zu Artikel 42a erarbeitet, und zum Zeitpunkt des Erscheinens dieser Ausgabe der Schweizerischen Ärztezeitung ist die Verordnung wahrscheinlich schon in der Vernehmlassung.

Aus den Protokollen der Diskussionen im Parlament wird deutlich, dass dieses vorab mit der Versichertenkarte an eine Vereinfachung administrativer Abläufe, insbesondere bei der Rechnungsstellung dachte. Es wird auch deutlich, dass mit Absatz 4 die Einführung einer «Gesundheitskarte» vorbereitet und generell E-Health in der Schweiz gefördert werden sollte. Die bisherigen Prinzipien des KVG (WZW-Kriterien, «tiers garant», hohe Wahlfranchisen und Freiwilligkeit von Managed-Care-Modellen) standen dabei nicht zur Debatte.

Die FMH akzeptiert im Grundsatz die Einführung einer Versichertenkarte und unterstützt die Erarbeitung praktikabler Lösungen [1]. Sie hat auch das Potential von E-Health generell erkannt und wird an der nächsten Ärztekammer das Projekt «Health Professional Card» als einen weiteren Baustein zur Umsetzung von E-Health und Erweiterung des heutigen Ärzteausweises zur Abstimmung vorlegen. Die FMH weist jedoch ausdrücklich auf die Probleme bei der Umsetzung der Versichertenkarte im allgemeinen und auf Probleme im Umgang mit medizinischen Daten auf der Karte im besonderen hin. Grundsätzlich muss mit jeder Lösung der Schutz des Patienten gewährleistet sein. Auch bezüglich administrativen Funktionen muss - gemeinsam mit der Ärzteschaft - eine in der Praxis wirklich umsetzbare und sinnvolle Lösung gefunden werden; die Ärztinnen und Ärzte* sind ja schliesslich diejenigen, die mit der Versichertenkarte arbeiten werden.

\section{Versichertenkarte und Gesundheitskarte}

Die Versichertenkarte wird vom Prinzip her im Bereich der obligatorischen Krankenversicherung von den Versicherern für jeden Versicherten ausgestellt. Sie ist Eigentum des jeweiligen Versicherers und könnte von diesem nach Ablauf zurückgefordert werden. Ein Patient könnte beispielsweise verschiedene Karten von unterschiedlichen Versicherern für Grund- und $\mathrm{Zu}$ satzversicherungen haben. Ein Versicherungswechsel würde die Ausstellung einer neuen Karte durch den neuen Versicherer auslösen.

Die FMH findet:

- Eine Versichertenkarte sollte ausschliesslich für administrative Zwecke eingesetzt werden.

- Das Konzept einer Versichertenkarte sollte ganz klar von dem einer Gesundheitskarte getrennt werden (schon nur aus Datenschutzgründen, aber auch aus Gründen der Praktikabilität und der Finanzierung).

- Eine Versichertenkarte (aber auch eine Gesundheitskarte) sollte selbst keine medizinischen Daten enthalten, da die Aktualität und Vollständigkeit nicht gewährleistet werden und die Daten bei Verlust ebenfalls verloren sein können.

\section{Art. 42a Versichertenkarte}

1 Der Bundesrat kann bestimmen, dass jede versicherte Person für die Dauer ihrer Unterstellung unter die obligatorische Krankenpflegeversicherung eine Versichertenkarte erhält. Diese enthält den Namen der versicherten Person und eine vom Bund vergebene Sozialversicherungsnummer.

2 Diese Karte mit Benutzerschnittstelle wird für die Rechnungsstellung der Leistungen nach diesem Gesetz verwendet.

3 Der Bundesrat regelt nach Anhören der interessierten Kreise die Einführung der Karte durch die Versicherer und die anzuwendenden technischen Standards.

4 Die Karte enthält im Einverständnis mit der versicherten Person persönliche Daten, die von dazu befugten Personen abrufbar sind. Der Bundesrat legt nach Anhören der interessierten Kreise den Umfang der Daten fest, die auf der Karte gespeichert werden dürfen. Er regelt den Zugriff auf die Daten und deren Bearbeitung. 
- Aufgrund der Freiwilligkeit, unsicherer Aktualität und Vollständigkeit sind medizinische Daten auf der Versichertenkarte, wie in Absatz 4 vorgesehen, nur von äusserst begrenztem Nutzen. Auch müsste sichergestellt werden, dass die medizinischen Daten im Notfall einfach, aber im Verlustfall oder bei Rückgabe an den Versicherer keinesfalls zugänglich sind.

- Das Recht des Patienten auf informationelle Selbstbestimmung und das Prinzip der Verhältnismässigkeit (bei der Speicherung von personenbezogenen Daten) sind unbedingt zu wahren.

Die Vermischung von Aspekten einer «Versichertenkarte» und einer «Gesundheitskarte» im KVG Art. 42a erachtet die FMH - was die Umsetzung anbelangt - als äusserst problematisch.

\section{Einsatz der Versichertenkarte - Verfahren zu Online-Abfragen}

Das Gesetz sieht den Einsatz der Versichertenkarte für die Rechnungsstellung der Leistungen nach KVG vor. In diesem Rahmen steht ein Verfahren zur Diskussion, bei dem mit Hilfe der Versichertenkarte halbjährlich die Leistungspflicht des Versicherers sowie aktuelle Versichertendaten und Deckungsinformationen bei den Versicherern online abgefragt werden. Analog zu Kreditkarten soll eine Nummer generiert werden, die auf der Rechnung erscheinen muss. Ein mögliches Ziel dieses Verfahrens könnte sein, eine medienbruchfreie Übernahme von Daten direkt von den Versicherern auf die Rechnung sicherzustellen und damit die Qualität der Versichertendaten auf der Rechnung zu verbessern (Vermeiden von Lese-, Schreib-, Übertragungsfehlern; Aktualität der Daten).

Die Meinung der FMH dazu:

- Die Tatsache, dass ein Patient einen Arzt konsultiert hat, unterliegt bereits der ärztlichen Schweigepflicht, d.h. es darf nicht bei einem Arztbesuch automatisch die Information über diesen Besuch an den Versicherer übermittelt werden.

- Es muss eine klare Trennung der Beziehung zwischen Patient und Versicherer sowie Patient und Arzt bestehen bleiben.

- Dem Patient muss die Möglichkeit offen gehalten werden, sich erst gegen Ende des Jahres dafür zu entscheiden, die Rechnung beim Versicherer einzureichen.

- Die Ziele des Einsatzes der Versichertenkarte sollten einvernehmlich, klar und überprüfbar definiert werden, und für die Ereichung dieser Ziele müssen dann angemessene Lösun- gen gefunden werden. Der Einsatz der Karte darf nicht zum Selbstzweck werden.

Die FMH geht von der Hypothese aus, dass mit dem Einsatz der Karte für die Rechnungsstellung der Leistungen eine Vereinfachung administrativer Abläufe und keine zusätzlichen administrativen Tätigkeiten angestrebt wurden. Demzufolge darf ein solches Verfahren keine administrativen Zusatzaufwände generieren, und es muss einen ausgewiesenen Nutzen mit sich bringen. Es müssen einfache, praktikable Lösungen für verschiedene Behandlungskonstellationen vorgesehen werden (z. B. Labor, Pathologie, Konsilien, Hausbesuche, Notfälle, beauftragte Leistungen, Belegarztverfahren, Patient hat Karte vergessen usw.), und es muss ein gleichwertiges Offline-Verfahren geben (die niedergelassenen Ärzte dürfen, u.a. aus Sicherheitsgründen, nicht dazu gezwungen werden online zu gehen).

Auch ist nicht klar, welche Konsequenzen das Nichtbestehen der Rückerstattungspflicht seitens des Versicherers haben sollte!

\section{Finanzierung}

Die Kosten-Nutzen-Analyse von Debold \& Lux [2] zeigt auf, dass der Nutzen der Versichertenkarte nur auf seiten der Versicherer liegt, Arztpraxen haben keinen Nutzen (im Gegensatz zu den Spitälern), nur Aufwand. Die von Bundesrat Couchepin am runden Tisch zugesicherte Finanzierungslösung wurde noch nicht erarbeitet. Die FMH fordert diese ein.

\section{Health Professional Cards (HPC), Institutionen- und andere Karten}

Der Einsatz der Versicherungskarte wird entsprechende Karten-«Pendants» bei den Leistungserbringern erforderlich machen, welche den Leistungserbringer als solchen elektronisch ausweisen (Authentisierung). Je nach Einsatz der Karte müssen diese mit unterschiedlichen Funktionalitäten ausgestattet sein («Health Professional Card» oder «elektronischer Heilberufeausweis» für die Ärzte, «Institutionenkarte» für administratives Personal in Spitälern und Arztpraxen bzw. Karten für medizinisches Hilfspersonal). Die FMH beabsichtigt, für die Ärzte eine HPC als elektronischen Ärzteausweis und davon abhängige Karten mit Teilfunktionalitäten herauszugeben. Sie wird die Interoperabilität mit der Versichertenkarte gewährleisten. Die Anforderungen sind sorgsam abzuwägen - beispielsweise hinterfragt die FMH die Forderung der qualifizierten elektronischen Signatur für Einträge auf der Versichertenkarte. 


\section{Fazit}

Die FMH weist in aller Deutlichkeit auf die kritischen Punkte bei der Einführung der Versichertenkarte hin und wird diese in der Vernehmlassung selbstverständlich ausführen. Die Berücksichtigung der erwähnten Probleme ist Voraussetzung für die Mitwirkung der Ärzte bei der Umsetzung dieses Projektes.

Für die Förderung der Verbreitung von E-Health in der Schweiz ist es ganz zentral, dass das Gesetz in sinnvoller und nutzbringender Weise umgesetzt wird. Alles andere wäre kontraproduktiv.

«Das Projekt steht und fällt mit der Akzeptanz der Anwender» [3] - diese «Weisheit» dürfte auf die Schweiz übertragbar sein!

\section{Literatur}

1 de Haller J. Versichertenkarte: Was denkt und was tut die FMH? Schweiz Ärztezeitung. 2006;87(33): 1399.

2 Debold \& Lux Beratungsgesellschaft für Informationssysteme und Organisation im Gesundheitswesen mbH. Die Versichertenkarte und der Aufbau einer Telematikinfrastruktur, Kosten-NutzenAnalyse. www.bag.admin.ch/themen/kranken versicherung/00305/00306/index.html?lang=de.

3 Bundesärztekammer. Deutschland: Diskussion um elektronische Gesundheitskarte. Schweiz Ärztezeitung. 2006;87(35):1513. 